\title{
NANOPARTICLES AS A BIORECOGNITION PLATFORM IN COMBINATION WITH LA-ICP-MS
}

\author{
1,2Marcela VLCNOVSKA, ${ }^{3}$ Aneta STOSSOVA, ${ }^{3}$ Michaela TVRDONOVA, ${ }^{2}$ Hana POLANSKA, \\ ${ }^{1,4}$ Marketa VACULOVICOVA, ${ }^{3}$ Tomas VACULOVIC, ${ }^{2}$ Michal MASARIK \\ ${ }^{1}$ Department of Chemistry and Biochemistry, Mendel University in Brno, Czech Republic, EU, \\ marcelavlcnovska@seznam.cz \\ ${ }^{2}$ Department of Pathological Physiology and ${ }^{3}$ Department of Chemistry Masaryk University, Brno, \\ Czech Republic, EU \\ ${ }^{4}$ Central European Institute of Technology, Brno University of Technology, Brno, Czech Republic, EU \\ https://doi.org/10.37904/nanocon.2019.8632
}

\begin{abstract}
Metal nanoparticles proved to be effective signaling tags in combination with laser ablation followed by inductively coupled plasma mass spectrometric detection. The surface may be suitably modified by various biorecognition elements such as antibodies or aptamers. Moreover, molecularly imprinted polymers can be prepared on their surface. In this study, the biorecognition capabilities of nanoparticles functionalized by antibodies and molecularly imprinted polymers were compared.
\end{abstract}

Keywords: Nanoparticles, LA-ICP-MS, antibodies, molecularly imprinted polymers

\section{INTRODUCTION}

Nanotechnology is focused on studying particles or materials in size ranges from 1 to $100 \mathrm{~nm}$ in at least one dimension. Such nanomaterials may be composed of organic substances, carbon or metals and their oxides. Nanoparticles can have different shapes, such as spheres, stars, rods, tubes, spirals, hollow cores, etc. Their shape, composition and size are responsible for their unique physical, chemical and biological properties compared to their equivalent in the macro scale. This is mainly due to the large ratio of particle surface to volume. The large active surface of particles can be used for chemical modification [1].

The surface of nanoparticles can be modified by binding to antibodies [2] or aptamers [3] used for specific recognition. The possibility of forming a molecularly imprinted polymer on the surface of magnetic particles was also presented. These particles can then also be used for specific recognition of imprinted structures in a complex sample, or isolation of target molecules [4].

Molecularly imprinted polymers (MIPs) are a technology of synthesis of polymers in the presence of a template molecule. After polymerization, the template molecule is removed to form selective recognition sites. The advantage of this method is that it can be used for analytes for which antibodies are not available, or their preparation is complicated. Not only small analytes but also proteins or whole cells can be imprinted [5].

Nanoparticles modified by antibody or molecularly imprinted polymers can be used for specific detection of analytes. The modified particles are used as a biorecognition tool. Laser ablation with inductively coupled plasma mass spectrometry (LA-ICP-MS) appears to be a suitable method of detection. LA-ICP-MS is a method of sample elemental analysis, which provides information not only about the elemental composition of the sample but also about spatial distribution $[6,7]$.

\section{MATERIAL}

Recombinant Human standard of protein p53 (ab43615) was purchased from Abcam, Cambridge, UK. An anti53 antibody DO-1 was delivered from Masaryk Memorial Cancer Institute in Brno. 
For the conjugation with antibodies and preparation of MIPs was used a commercial kit of europium nanoparticles Europium Conjugation Kit from Expedeon, San Diego, USA.

Dopamine hydrochloride Sigma-Aldrich (USA) was used as a functional monomer for MIP preparation

\section{METHODS}

\subsection{Conjugates preparation}

Conjugation of 200nm EuNPs with anti-p53 antibody was performed according to the kit manufacturer's protocol. Conjugate was afterwards used for dot-blot analysis.

\subsection{MIPs}

$10 \mu \mathrm{l}$ of $200 \mathrm{~nm}$ EuNPs without antibody was washed 3 times by $200 \mu \mathrm{l} 20 \mathrm{mM}$ Tris-HCl pH 8.5 and centrifuged for 10 minutes, $17000 \mathrm{rcf}$. After that $600 \mu \mathrm{l}$ of lysozyme $0.2 \mathrm{mg} / \mathrm{ml}$ was pipetted. The mixture was gently mixed on Multi RS-60 (Biosan, Latvia), $10 \mathrm{rpm}$. Then $100 \mu \mathrm{l}$ of $3,5 \mathrm{mg} / \mathrm{ml}$ dopamine was added to create a polymer on the surface of nanoparticles. Polymerization lasted for 15 hours. Subsequently, the template of lysozyme was washed out 3 times with $200 \mu \mathrm{l} 10 \% \mathrm{HAc}$ and finally $200 \mu \mathrm{l} 20 \mathrm{mM}$ Tris-HCl.

NIPs (non-imprinted polymers) were prepared as a negative control was in the same way. The only difference was the addition of water instead of the lysozyme template.

These modified particles were used for dot-blot analysis.

\subsection{Dot-blot}

The Dot-blot analysis with following LA-ICP-MS was performed according to Tvrdonova et al. 2019 [7] Briefly, on the activated PVDF membrane (Bio-Rad, USA) were applied the samples of protein p53 ( $0.5 \mu \mathrm{l}$ ) or lysozyme $(1 \mu \mathrm{l})$ in different concentration. After 30 minutes the membrane was blocked by blocking buffer also for 30 minutes. Subsequently, incubation with modified nanoparticles was carried out for 1 hour. Finally, the membranes were three times washing with PBS-T for 5 min and then analyzed by LA-ICP-MS.

\section{RESULTS AND DISCUSSION}

\subsection{Antibody-modified nanoparticles}

The conventional immunoassay is a well-established technique; however, new and improved alternatives have been still searched. From this point of view, a powerful elemental analytical method such as LA-ICP-MS may be beneficial, therefore in this study, an anti-p53 antibody was labeled by $200 \mathrm{~nm}$ europium nanoparticles and employed as a biorecognition element in the dot-blot analysis of p53. As shown in Figure 1, the detection of the target analyte at the nanogram level was enabled.

Figure 1 Dot blot analysis of p53 protein using anti-53 antibody labeled by $200 \mathrm{~nm}$ europium nanoparticle and detected by LA-ICP-MS

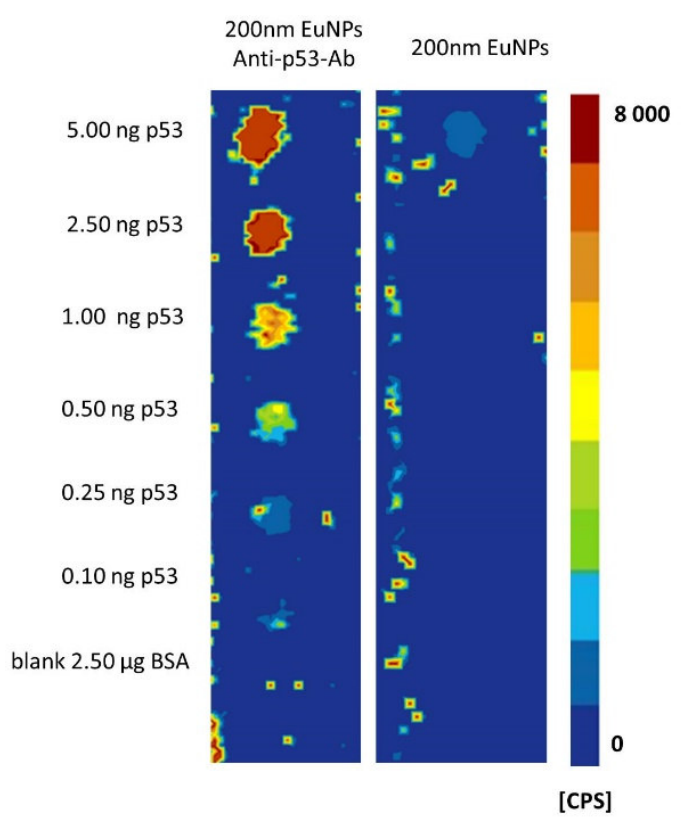




\subsection{MIPs on the surface of nanoparticles}

Due to some disadvantages of antibodies as biorecognition elements which include relatively poor chemical stability, high price, unavailability for certain analytes or ambiguity in specific chemical antibody-antigen interaction mechanisms, MIPs may serve as an alternative overcoming the above-mentioned obstacles. Therefore, we decided to combine the power of LA-ICP-MS in sensitivity of detection, the high metal signal provided by metal-based nanoparticles due to the content of the high number of metal atoms, and benefit provided by biorecognition abilities of MIP surface.

Figure 2 shows the Eu signals of nanoparticles with surface imprinted by the polydopamine layer specifically developed to selectively recognize lysozyme as a target. Lysozyme was selected due to its relatively small molecular mass because the imprinting efficiency is higher for smaller templates. For imprinting of larger molecules, epitope imprinting is commonly utilized. As shown in Figure 2, non-imprinted polymeric layer (NIP) provides a significantly lower signal. To verify the approach, iron oxide magnetic particles were also tested (Figure 3).

Figure 2 Dot blot analysis of lysozyme using polydopamine MIP on the surface of Eu nanoparticles $(200 \mathrm{~nm})$ and detected by LA-ICP-MS

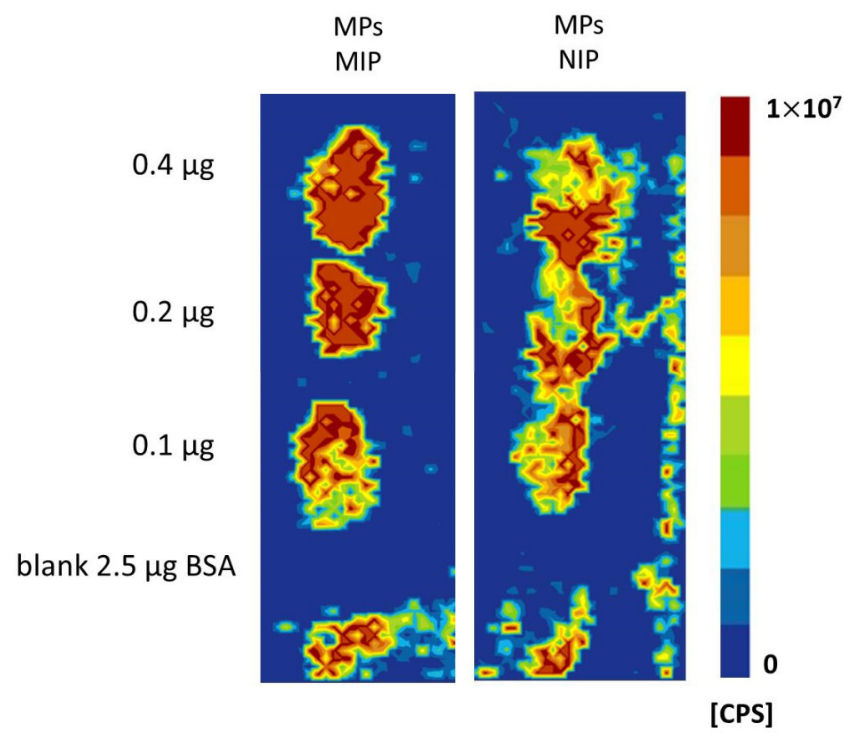

Figure 3 Dot blot analysis of lysozyme protein using polydopamine MIP on the surface of magnetic iron oxide nanoparticles $(1 \mu \mathrm{m})$ and detected by LA-ICP-MS

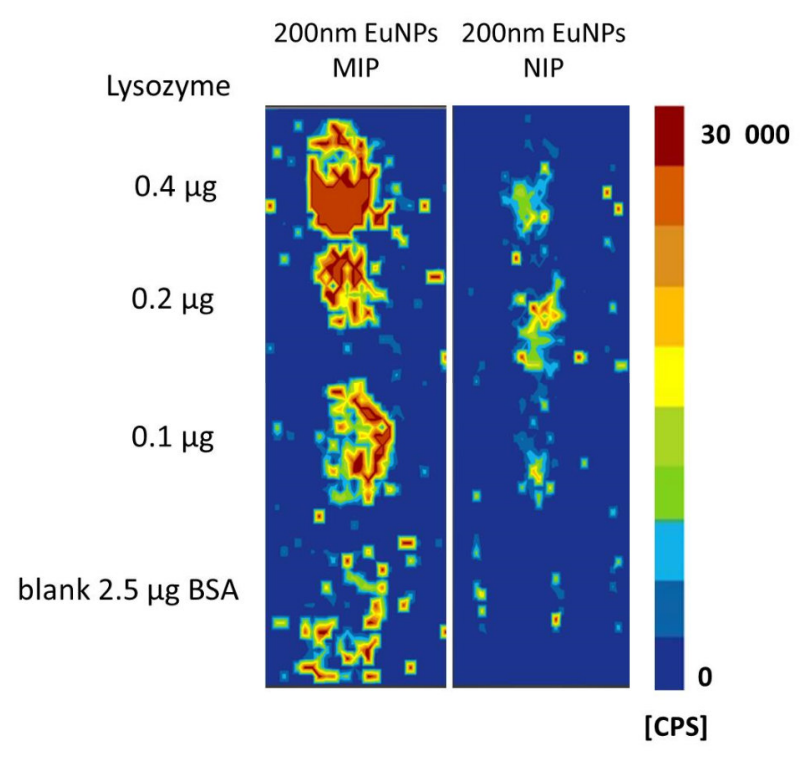

As shown in Figure 3, the signal intensities were significantly higher compared to signal provided by Eu nanoparticles, however the iron oxide nanoparticles are significantly higher in diameter $(1 \mu \mathrm{m})$, therefore also the nonspecific sorption is higher. However, there is still a satisfactory difference between MIP and NIP particles. To improve the performance of the assay, gold nanoparticles can be used, polymer providing better imprinting properties (e.g. methacrylate) is applicable, epitope imprinting approach would be beneficial, and finally, nanoparticle surface blocking strategies (similar to those used in conventional immunoassay analyses) may be tested and optimized.

\section{CONCLUSION}

In this study, MIPs as biorecognition elements were synthesized on the surface of metal-based nanoparticles and used in dot blot analysis of proteins with coupling to LA-ICP-MS detection. The use of MIPs as the 
alternative to antibodies may be beneficial in cases when antibodies are not available or provide undesirable cross-reactivity. The combination of MIPs, metal particles and LA-ICP-MS is a promising technique complementing the existing family of bioanalytical techniques.

\section{ACKNOWLEDGEMENTS}

\section{The research was financially supported by the Czech Science Foundation (GACR 17-12774S) and by the Grant Agency of Masaryk University (MUNI/C/0003/2019).}

\section{REFERENCES}

[1] JEEVANANDAM, J. et al. Review on nanoparticles and nanostructured materials: history, sources, toxicity and regulations. Beilstein Journal of Nanotechnology. 2018. vol. 9, pp. 1050-1074.

[2] RYAN, S. et al. Single-Domain Antibody-Nanoparticles: Promising Architectures for Increased Staphylococcus aureus Detection Specificity and Sensitivity. Bioconjugate Chemistry. 2009. vol. 20, no. 10, pp. 1966-1974.

[3] PAVLOV, V. et al. Aptamer-functionalized Au nanoparticles for the amplified optical detection of thrombin. Journal of the American Chemical Society. 2004. vol. 126, no. 38, pp. 11768-11769.

[4] ZEMANKOVA, et al. Combinations of capillary electrophoresis-UV/Vis and molecularly imprinted polymers for detection of phytoestrogens. In MendelNet 2018: $25^{\text {th }}$ International Undergraduate and Postgraduate Students Conference. Brno: MENDEL UNIV BRNO, 2018, pp. 553-556.

[5] VANECKOVA, T. et al. CdS quantum dots-based immunoassay combined with particle imprinted polymer technology and laser ablation ICP-MS as a versatile tool for protein detection. Scientific Reports. 2019. vol. 9 (11840)

[6] POZEBON, D. et al. Recent applications of laser ablation inductively coupled plasma mass spectrometry (LA-ICPMS) for biological sample analysis: a follow-up review. Journal of Analytical Atomic Spectrometry. 2017. vol. 32, no. 5, pp. 890-919.

[7] TVRDONOVA, M. et al. Gold nanoparticles as labels for immunochemical analysis using laser ablation inductively coupled plasma mass spectrometry. Analytical and Bioanalytical Chemistry. 2019. vol. 411, no. 3, pp. 559-564 